\title{
CONSTANT ISOTROPIC SUBMANIFOLDS WITH 4-PLANAR GEODESICS
}

\author{
JIN SUK PAK AND KUNIO SAKAMOTO
}

\begin{abstract}
Let $f$ be an isometric immersion of a Riemannian manifold $M$ into $\bar{M}$. We prove that if $f$ is constant isotropic, 4-planar geodesic and $\bar{M}$ is a Euclidean sphere, then $M$ is isometric to one of compact symmetric spaces of rank equal to one and $f$ is congruent to a direct sum of standard minimal immersions. We also determine constant isotropic, 4-planar geodesic, totally real immersions into a complex projective space of constant holomorphic sectional curvature.
\end{abstract}

0. Introduction. In [15], O'Neill studied isotropic immersions and showed that if the difference between the sectional curvatures of the submanifold and the ambient manifold is constant for any plane section tangent to the submanifold, then the codimension must be high compared with the dimension of the submanifold unless the immersion is totally geodesic or umbilical. The class of (constant) isotropic immersions (for instance, into a sphere) seems to be too large to classify them. For example, the composition of two isotropic immersions is also isotropic and for two given isotropic immersions $f_{1}$ and $f_{2}$ into $S^{p}(1)$ and $S^{q}(1)$, respectively, their "direct sum" $\left(\cos \theta f_{1}, \sin \theta f_{2}\right)$ into $S^{p+q+1}(1)$ is also isotropic for any $\theta$. Furthermore, it is well known that there are many constant isotropic immersions in equivariant isometric immersions of homogeneous spaces.

An isometric immersion $f: M \rightarrow \bar{M}$ is said to be 4-planar geodesic if, for each geodesic $\tau$ of $M$, the curve $f \circ \gamma$ is locally contained in a 4-dimensional totally geodesic submanifold of $M$. The class of 4-planar geodesic immersions (into a sphere) is also large. At least, an isometric immersion of a surface into a 4dimensional Riemannian manifold is always 4-planar geodesic.

In this paper, we try to classify immersions in the intersection of these classes. In particular, we are able to classify constant isotropic, 4-planar geodesic (resp. and totally real) immersions of connected, complete Riemannian manifolds into a unit sphere (resp. complex projective space of constant holomorphic sectional curvature $4)$.

In $\S 1$, we give basic equations used later and some definitions. In $\S 2$, we prove that if $f$ is a constant isotropic, 4-planar geodesic (resp. and totally real) immersion into a real space form (resp. $C P^{q}(c)$ ), then $f$ is a helical immersion of order $\leq 4$. Here we recall that an isometric immersion $f: M \rightarrow \bar{M}$ is called a helical immersion

Received by the editors April 3, 1987.

1980 Mathematics Subject Classification (1985 Revision). Primary 53C40.

Key words and phrases. Second fundamental forms, isotropic and 4-planar geodesic immersions, totally real immersions.

Work done under partial support by a research grant of Korea Science and Engineering Foundation. 
of order $d$ if, for each geodesic $\gamma$ of $M$, the curve $f \circ \gamma$ in $\bar{M}$ has constant Frenet curvatures which are independent of the chosen geodesic and the osculating order is equal to $d[\mathbf{2 0}]$. In [8], it was shown that if $f: M \rightarrow$ (real space form) is an isometric immersion with every geodesic being of order $d$ (even) and the Frenet curvatures $\kappa_{1}, \ldots, \kappa_{(d / 2)-1}$ are constant (independent of the chosen geodesic), then $f$ is a helical immersion of order $d$. However, it should be noticed that our assumption "4-planar geodesic and constant isotropic" is weaker than [8] when $d=4$ and the ambient manifold is a real space form. In $\S 3$, we generalize the results about helical, minimal immersions of order $\leq 4$ into spheres $[\mathbf{2 1}, \mathbf{2 2}]$ to not necessarily minimal ones and show that the submanifolds are compact symmetric spaces of rank one. $\mathrm{T}$. Tsukada [24] proved that every helical immersion of a compact symmetric space of rank one into a sphere is congruent to a direct sum of some standard minimal immersions and calculated the order of the direct sum (see also [7]). Making use of his result, we classify constant isotropic, 4-planar geodesic immersions into a unit sphere (Theorem 3.6). In contrast with a real space form, we should note that a curve of order $d$ with constant Frenet curvatures in a complex space form is not always contained in a $d$-dimensional totally geodesic submanifold. We can see this fact in the classification of isotropic, totally real submanifolds with parallel second fundamental forms in $C P^{q}(c)$, which was done in [11]. By using [11], we classify constant isotropic, 4-planar geodesic, totally real immersions into $C P^{q}(4)$ (Theorem 3.11). In $\S 4$, we show that an example given in the study of stationary 2-type surfaces in a sphere [1] is isotropic and 5-planar geodesic (but not 4-planar geodesic). This shows that it is impossible to replace "4-planar geodesic" by "5planar geodesic" in Theorems 2.6 and 2.7 (see also Remark in $§ 2$ ).

1. Basic equations. Let $f: M \rightarrow \bar{M}$ be an isometric immersion of an $n$ dimensional Riemannian manifold $M$ into an $m$-dimensional Riemannian manifold $\bar{M}$. We denote by $\nabla$ and $\bar{\nabla}$ the Riemannian connections of $M$ and $\bar{M}$, respectively. The second fundamental form, Weingarten map corresponding to a normal vector $\xi$ and the normal connection is denoted by $H, A_{\xi}$ and $\nabla^{\perp}$, respectively.

For vector fields $X$ and $Y$ tangent to $M$, Gauss and Weingarten's equations are given by

$$
\begin{gathered}
\bar{\nabla}_{X} Y=\nabla_{X} Y+H(X, Y), \\
\bar{\nabla}_{X} \xi=-A_{\xi} X+\nabla_{X}^{\perp} \xi,
\end{gathered}
$$

where $\xi$ is a normal vector field. In the sequel, we will deal with the case where the ambient manifold $\bar{M}$ is a simply connected, complete Riemannian manifold $\bar{M}(c)$ of constant curvature $c$ (i.e., $S^{m}(c), E^{m}$ or $D^{m}(c)$ ) or a complex projective space $C P^{q}(c)$ of constant holomorphic sectional curvature $c$, where $m=2 q$. Let $R$ be the curvature tensor of $M$ and $D$ the van der Waerden-Bortolotti connection. When $\bar{M}=\bar{M}(c)$, the structure equations of Gauss, Codazzi and Ricci are respectively given by

$$
\begin{gathered}
R(X, Y) Z=c\{\langle Y, Z\rangle X-\langle X, Z\rangle Y\}+A_{H(Y, Z)} X-A_{H(X, Z)} Y \\
\left(D_{X} H\right)(Y, Z)-\left(D_{Y} H\right)(X, Z)=0 \\
R^{\perp}(X, Y) \xi=H\left(X, A_{\xi} Y\right)-H\left(A_{\xi} X, Y\right)
\end{gathered}
$$


where $R^{\perp}$ denotes the curvature tensor of the connection $\nabla^{\perp}$. Let $J$ be the complex structure of $C P^{q}(c)$. Denote by $\operatorname{Proj}_{T M}$ and $\operatorname{Proj}_{N M}$ the projections of $T C P^{q}(c)$ to the tangent bundle $T M$ and the normal bundle $N M$, respectively and put $\tilde{J}=$ $\operatorname{Proj}_{T M} \circ J\left|T M, J_{N}=\operatorname{Proj}_{N M} \circ J\right| T M, J_{T}=\operatorname{Proj}_{T M} \circ J \mid N M$ and $J^{\perp}=\operatorname{Proj}_{N M} \circ$ $J \mid N M$. Then we can write

$$
J X=\tilde{J} X+J_{N} X, \quad J \xi=J_{T} \xi+J^{\perp} \xi
$$

for every tangent vector $X$ and normal vector $\xi$. Using these tensors, we can write structure equations of $f: M \rightarrow C P^{q}(c)$ as

$$
\begin{gathered}
R(X, Y) Z=(c / 4)\{\langle Y, Z\rangle X-\langle X, Z\rangle Y+\langle\tilde{J} Y, Z\rangle \tilde{J} X \\
\quad-\langle\tilde{J} X, Z\rangle \tilde{J} Y-2\langle\tilde{J} X, Y\rangle \tilde{J} Z\} \\
+A_{H(Y, Z)} X-A_{H(X, Z)} Y \\
\left(D_{X} H\right)(Y, Q)-\left(D_{Y} H\right)(X, Z) \\
=(c / 4)\left\{\langle\tilde{J} Y, Z\rangle J_{N} X-\langle\tilde{J} X, Z\rangle J_{N} Y-2\langle\tilde{J} X, Y\rangle J_{N} Z\right\}, \\
R^{\perp}(X, Y) \xi=(c / 4)\left\{\left\langle J_{N} Y, \xi\right\rangle J_{N} X-\left\langle J_{N} X, \xi\right\rangle J_{N} Y-2\langle\tilde{J} X, Y\rangle J^{\perp} \xi\right\} \\
+H\left(X, A_{\xi} Y\right)-H\left(A_{\xi} X, Y\right) .
\end{gathered}
$$

We easily see that $J_{N}=J_{T}=0$ if and only if $M$ is a Kaehler submanifold. If $\tilde{J}=0$, then $f: M \rightarrow C P^{q}(c)$ is said to be totally real and equations $(1.3)^{\prime}$ and $(1.4)^{\prime}$ reduce to the same as (1.3) and (1.4), respectively. Moreover, in the case where $f$ is totally real, we have

$$
\left\langle J_{N} Y, H(X, Z)\right\rangle=\left\langle J_{N} Z, H(X, Y)\right\rangle
$$

for every $X, Y, Z \in T_{x} M$, which is derived from $\bar{\nabla} J=0$.

A curve $\tau: I \rightarrow \bar{M}$ defined on an open integral $I$ is said to be $d$-planar if there exist an open interval $I_{s}\left(s \in I_{s} \subset I\right)$ and a $d$-dimensional totally geodesic submanifold $P_{s}$ of $\bar{M}$ for each $s \in I$ such that $\tau\left(I_{s}\right) \subset P_{s}$. An isometric immersion $f: M \rightarrow \bar{M}$ is said to be $d$-planar geodesic if $\tau=f \circ \gamma$ is $d$-planar for every geodesic $\gamma: I \rightarrow M$. 1-planar geodesic immersions are totally geodesic. 2-planar geodesic immersions were studied in [9 and 19] and 3-planar geodesic immersions were studied in [14 and 17].

An isometric immersion $f: M \rightarrow \bar{M}$ is said to be $(\lambda$-) isotropic if

$$
\|H(X, X)\|=\lambda(\pi(X))
$$

for every $X \in U M$, where $\pi: U M \rightarrow M$ is the projection of the unit tangent bundle of $M$ and $\lambda$ is a function defined on $M$ (cf. [15]). Moreover, if $\lambda$ is constant, then the immersion is said to be constant isotropic. We easily see that $f$ is isotropic if and only if

$$
\langle H(X, X), H(X, Y)\rangle=0
$$

for any orthogonal vectors $X$ and $Y$ tangent to $M$ at every point. Equation (1.7) implies that

$$
\langle H(X, X), H(Y, Y)\rangle+2\|H(X, Y)\|^{2}=\lambda^{2}
$$

for orthonormal vectors $X$ and $Y$. By this equation and $(1.3)^{\prime}$, we can prove (cf. [18]) 
PROPOSITION 1.1. Let $M$ be a connected, complete Kaehler manifold of complex dimension $r \geq 2$. If $f: M \rightarrow C P^{q}(c)$ is an isotropic Kaehler immersion, then $M$ is isometric to $C P^{r}(c / k)$ and $f$ is equivalent to the kth complex Veronese immersion for some positive integer $k$.

In the following section, we shall need a Codazzi equation of the form (1.4) and hence the assumption that $f: M \rightarrow C P^{q}(c)$ is totally real.

2. Frenet curvatures. Let $f: M \rightarrow \bar{M}(c)$ (resp. $C P^{q}(c)$ ) be a constant isotropic (resp. totally real, constant isotropic) immersion. We always assume that $n \geq 2$. Let $\gamma$ be any unit speed geodesic of $M, x=\gamma(0)$ and $X=\dot{\gamma}(0)$.

LEMMA 2.1. For the curve $\tau=f \circ \gamma$ in $\bar{M}$, we have

$$
\begin{aligned}
& \bar{\nabla}_{X} \dot{\tau}=H(X, X), \\
& \bar{\nabla}_{X}^{2} \dot{\tau}=-\lambda^{2} X+(D H)\left(X^{3}\right), \\
& \bar{\nabla}_{X}^{3} \dot{\tau}=-\lambda^{2} H(X, X)+\left(D^{2} H\right)\left(X^{4}\right), \\
& \bar{\nabla}_{X}^{4} \dot{\tau}=\lambda^{4} X-A_{\left(D^{2} H\right)\left(X^{4}\right)} X-\lambda^{2}(D H)\left(X^{3}\right)+\left(D^{3} H\right)\left(X^{5}\right),
\end{aligned}
$$

where we have put $(D H)\left(X^{3}\right)=(D H)(X, X, X)$ and so on.

PROOF. The first two equations are easily derived from (1.1), (1.2) and (1.8). Let $Y$ be any unit tangent vector orthogonal to $X$ at $x$. Extend them to orthonormal, local vector fields $X^{*}$ and $Y^{\circ}$ around $x$ such that $\nabla X^{*}=\nabla Y^{\circ}=0$ at $x$. Then from (1.8) and (1.9)

$$
\begin{aligned}
0 & =Y \cdot\left\|H\left(X^{*}, X^{*}\right)\right\|^{2}=2\langle(D H)(Y, X, X,), H(X, X)\rangle, \\
0 & =X \cdot\left\langle H\left(X^{*}, X^{*}\right), H\left(X^{*}, Y^{*}\right)\right\rangle \\
& =\left\langle(D H)\left(X^{3}\right), H(X, Y)\right\rangle+\langle H(X, X),(D H)(X, X, Y)\rangle,
\end{aligned}
$$

from which we have

$$
\langle H(X, X),(D H)(X, X, Y)\rangle=\left\langle(D H)\left(X^{3}\right), H(X, Y)\right\rangle=0 .
$$

Since $\left\langle H(X, X),(D H)\left(X^{3}\right)\right\rangle=0,(2.1)$ holds for any tangent vectors $X$ and $Y$. Making use of (2.1), we can easily show the last two equations. Q.E.D.

If $\lambda=0$, then $f$ is totally geodesic. Thus we have only to consider the case $\lambda \neq 0$.

LEMMA 2.2. Assume that $f$ is 4-planar geodesic. Then the function $\mu: U M \rightarrow$ $[0, \infty]$ defined by

$$
\mu(X)=\left\|(D H)\left(X^{3}\right)\right\| / \lambda
$$

is constant on each fiber $U_{x} M$.

PROOF. We shall prove that the derivative of $\mu^{2} \mid U_{x} M$ vanishes. If $\mu^{2}(X)=0$, then $\mu^{2}$ attains the minimum at $X$ and hence the derivative vanishes at $X$. Let $S_{1}=\{X \in U M ; \mu(X) \neq 0\}$ and $Y$ be an arbitrary tangent vector orthogonal to $X \in S_{1} \cap U_{x} M$. Identify $Y$ with a vector tangent to $U_{x} M$ at $X$. Then it suffices to show that $Y \cdot \mu^{2}=0$ which is equivalent to

$$
\left\langle(D H)\left(X^{3}\right),(D H)(X, X, Y)\right\rangle=0 .
$$


Define $\tau_{1}(X), \tau_{2}(X)$ and $\tau_{3}(X)$ by

$$
\tau_{1}(X)=X, \quad \tau_{2}(X)=H(X, X) / \lambda, \quad \tau_{3}(X)=(D H)\left(X_{3}\right) / \lambda \mu
$$

for $X \in S_{1}$ and $\nu: S_{1} \rightarrow[0, \infty]$ by

$$
\nu(X)=\left\|\bar{\nabla}_{X} \tau_{3}\left(\dot{\gamma}_{X}\right)+\mu \tau_{2}(X)\right\|,
$$

where $\gamma_{X}$ is the unit speed geodesic tangent to $X$ at $\gamma(0)$. Using (1.2) and the definition of $\mu$, we have

$$
\begin{aligned}
\bar{\nabla}_{X} \tau_{3}\left(\dot{\gamma}_{X}\right)+\mu \tau_{2}(X)= & \mu \tau_{2}(X)-\left(1 / \lambda^{2} \mu^{2}\right)\left\langle(D H)\left(X^{3}\right),\left(D^{2} H\right)\left(X^{4}\right)\right\rangle \tau_{3}(X) \\
& +(1 / \lambda \mu)\left(D^{2} H\right)\left(X^{4}\right) .
\end{aligned}
$$

If $\nu(X) \neq 0$, then $\tau_{4}(X)$ is defined by

$$
\tau_{4}(X)=\left\{\bar{\nabla}_{X} \tau_{3}\left(\dot{\gamma}_{X}\right)+\mu \tau_{2}(X)\right\} / \nu .
$$

We have defined orthonormal vectors $\tau_{1}(X), \tau_{2}(X), \tau_{3}(X)$ and $\tau_{4}(X)$ for $X \in\{X \in$ $U M ; \mu(X) \neq 0, \nu(X) \neq 0\}$. Since $f$ is 4-planar geodesic, there exist a 4-dimensional totally geodesic submanifold $P$ of $\bar{M}$ and an open interval $I(\ni 0)$ such that $\tau_{X}(I) \subset$ $P$, where $\tau_{X}=f \circ \gamma_{X}$. Thus $\left\{\tau_{i}(X)\right\}, i=1,2,3,4$, forms the Frenet frame of $\tau_{X}$ and $\operatorname{span} T_{\pi(X)} P$ at $\pi(X)$.

Let $X \in S_{1} \cap U_{x} M$ be arbitrarily fixed. Since (2.1) implies

$$
\left\langle\left(D^{2} H\right)\left(X^{4}\right), H(X, Y)\right\rangle+\left\langle(D H)\left(X^{3}\right),(D H)(X, X, Y)\right\rangle=0,
$$

(2.2) is equivalent to

$$
\left\langle\left(D^{2} H\right)\left(X^{4}\right), H(X, Y)\right\rangle=0
$$

for every tangent vector $Y$ orthogonal to $X$. Suppose that $\nu(X) \neq 0$. Taking the fact that $\tau_{2}(X), \tau_{3}(X)$ and $\tau_{4}(X)$ are normal to $M$ into account, we see that $Y$ is orthogonal to $T_{x} P$. Since $\bar{\nabla}_{X}{ }^{4} \tau_{X}$ is tangent to $P$, we obtain (2.6) by the last equation of Lemma 2.1. Next suppose that $\nu(X)=0 .\left(D^{2} H\right)\left(X^{4}\right)$ is a linear combination of $\tau_{2}(X)$ and $\tau_{3}(X)$ because of (2.4). Thus (2.6) is derived from (1.8) and (2.1). Q.E.D.

REMARK. In the above proof. We used conditions that $f$ is constant isotropic and 4-planar geodesic to prove (2.6). However, we can not replace "4-planar geodesic" by "5-planar geodesic" because we have an example of constant isotropic, 5 -planar geodesic immersions such that $\mu$ depends on $X \in U_{x} M$. See $\S 4$.

By Lemma 2.2, $\mu$ can be considered as a function on $M$, which is also denoted by $\mu$. Let $f$ be 4 -planar geodesic.

LEMMA 2.3. The function $\mu$ is constant on $M$.

ProOF. It suffices to show that the derivative of $\mu^{2}$ vanishes on $S_{2}=\{x \in$ $M ; \mu(x) \neq 0\}$. Let $x \in S_{2}$ and $Y \in U_{x} M$ be arbitrarily fixed. Since $n \geq 2$ and

$$
Y \cdot \mu^{2}=\left(2 / \lambda^{2}\right)\left\langle\left(D^{2} H\right)\left(Y, X^{3}\right),(D H)\left(X^{3}\right)\right\rangle,
$$

we shall prove

$$
\left\langle\left(D^{2} H\right)\left(Y, X^{3}\right),(D H)\left(X^{3}\right)\right\rangle=0
$$

for a unit tangent vector $X$ orthogonal to $Y$. In order to prove (2.7), we must show

$$
\left\langle\left(D^{2} H\right)\left(Y, X^{3}\right),(D H)\left(X^{3}\right)\right\rangle=\left\langle\left(D^{2} H\right)\left(X^{3}, Y\right),(D H)\left(X^{3}\right)\right\rangle .
$$


Assume that (2.8) holds. From (2.2), we have

$$
\left\langle\left(D^{2} H\right)\left(X^{4}\right),(D H)(X, X, Y)\right\rangle+\left\langle(D H)\left(X^{3}\right),\left(D^{2} H\right)\left(X^{3}, Y\right)\right\rangle=0
$$

and hence

$$
\left\langle\left(D^{2} H\right)\left(Y, X^{3}\right),(D H)\left(X^{3}\right)\right\rangle=-\left\langle\left(D^{2} H\right)\left(X^{4}\right),(D H)(X, X, Y)\right\rangle .
$$

Moreover, from (2.6)

$$
\left\langle\left(D^{3} H\right)\left(X^{5}\right), H(X, Y)\right\rangle+\left\langle\left(D^{2} H\right)\left(X^{4}\right),(D H)(X, X, Y)\right\rangle=0 .
$$

Therefore (2.7) is equivalent to $\left\langle\left(D^{3} H\right)\left(X^{5}\right), H(X, Y)\right\rangle=0$. Suppose that $\nu(X) \neq$ 0 . Then $\left\langle\tau_{i}(X), H(X, Y)\right\rangle=0$ for $i=2,3,4$ because of (1.8), (2.1) and (2.6). Therefore $\left\langle\bar{\nabla}_{X}^{4} \dot{\tau}_{X}, H(X, Y)\right\rangle=0$ because $f$ is 4 -planar geodesic. By the last equation of Lemma 2.1 , we have (2.7). If $\nu(X)=0$, then (2.7) follows from (2.1), (2.2), (2.4) and (2.9).

To complete the proof, we have only to prove (2.8) for every orthonormal vector $X$ and $Y$ at $x \in S_{2}$. From Ricci's identity

$$
\left(D^{2} H\right)\left(Y, X^{3}\right)-\left(D^{2} H\right)\left(X, Y, X^{2}\right)=R^{\perp}(Y, X) H(X, X)-2 H(R(Y, X) X, X)
$$

and (2.1), we have

$$
\begin{gathered}
\left\langle\left(D^{2} H\right)\left(Y, X^{3}\right)-\left(D^{2} H\right)\left(X, Y, X^{2}\right),(D H)\left(X^{3}\right)\right\rangle \\
=\left\langle R^{\perp}(Y, X) H(X, X),(D H)\left(X^{3}\right)\right\rangle .
\end{gathered}
$$

If the ambient manifold $\bar{M}$ is $\bar{M}(c)$ of constant sectional curvature $c$, then the right-hand side of (2.11) vanishes by Ricci's structure equation (1.5), (1.8) and (2.1). Next, suppose that $\bar{M}=C P^{q}(c)$ and $f$ is totally real. Substituting $(1.5)^{\prime}$ into the right-hand side of $(2.11)$, we obtain

$$
\begin{aligned}
& \left\langle\left(D^{2} H\right)\left(Y, X^{3}\right)-\left(D^{2} H\right)\left(X, Y, X^{2}\right),(D H)\left(X^{3}\right)\right\rangle \\
& =(c / 4)\left\{\langle J X, H(X, X)\rangle\left\langle J Y,(D H)\left(X^{3}\right)\right\rangle-\langle J Y, H(X, X)\rangle\left\langle J X,(D H)\left(X^{3}\right)\right\rangle\right\},
\end{aligned}
$$

where we have used (2.1). Let $\gamma_{X}$ be the geodesic as before and $P$ be a 4-dimensional totally geodesic submanifold which contains $\tau_{X}(I)$ for some open interval $I(\ni 0)$, where $I$ is taken small enough to satisfy $\tau_{X}(I) \subset S_{2}$. Note that $\tau_{1}(\dot{\gamma}), \tau_{2}(\dot{\gamma})$ and $\tau_{3}(\dot{\gamma})$ are tangent to $P$ on $I$. Thus if $P$ is totally real, then $J X$ is orthogonal to $H(X, X)$ and $(D H)\left(X^{3}\right)$, and hence the right-hand side of $(2.1)$ vanishes. So we assume that $P$ is a complex submanifold in $C P^{q}(c)$. Define a complex subspace $\Pi_{s}$ in $T_{\gamma(s)} P$ by

$$
\Pi_{s}=\operatorname{Span}\left\{\dot{\gamma}, J \dot{\gamma}, H(\dot{\gamma}, \dot{\gamma}), J H(\dot{\gamma}, \dot{\gamma}),(D H)\left(\dot{\gamma}^{3}\right), J(D H)\left(\dot{\gamma}^{3}\right)\right\}
$$

for each $s \in I$. Clearly, $\operatorname{dim} \Pi_{s}=4$ for every $s$. Thus there exist functions $\alpha_{1}, \ldots, \alpha_{5}$ on $I$ such that $\left(\alpha_{1}(s), \ldots, \alpha_{5}(s)\right) \neq 0$ for each $s$ and

$$
\alpha_{1} \dot{\gamma}+\alpha_{2} J \dot{\gamma}+\alpha_{3} H(\dot{\gamma}, \dot{\gamma})+\alpha_{4} J H(\dot{\gamma}, \dot{\gamma})+\alpha_{5}(D H)\left(\dot{\gamma}^{3}\right)=0 .
$$


Making use of (1.6), we have

$$
\begin{aligned}
& \alpha_{1}-\langle J \dot{\gamma}, H(\dot{\gamma}, \dot{\gamma})\rangle \alpha_{4}=0, \\
& \alpha_{2}+\langle J \dot{\gamma}, H(\dot{\gamma}, \dot{\gamma})\rangle \alpha_{3}+\left\langle J \dot{\gamma},(D H)\left(\dot{\gamma}^{3}\right)\right\rangle \alpha_{5}=0, \\
& \langle J \dot{\gamma}, H(\dot{\gamma}, \dot{\gamma})\rangle \alpha_{2}+\lambda^{2} \alpha_{3}=0, \\
& -\langle J \dot{\gamma}, H(\dot{\gamma}, \dot{\gamma})\rangle \alpha_{1}+\lambda^{2} \alpha_{4}+\left\langle J H(\dot{\gamma}, \dot{\gamma}),(D H)\left(\dot{\gamma}^{3}\right)\right\rangle \alpha_{5}=0, \\
& \left\langle J Y^{\cdot}, H(\dot{\gamma}, \dot{\gamma})\right\rangle \alpha_{4}=0, \\
& \left\langle J Y^{\cdot}, H(\dot{\gamma}, \dot{\gamma})\right\rangle \alpha_{3}+\left\langle J Y^{\cdot},(D H)\left(\dot{\gamma}^{3}\right)\right\rangle \alpha_{5}=0, \\
& \left\langle J Y^{\cdot}, H(\dot{\gamma}, \dot{\gamma})\right\rangle \alpha_{2}+\left\langle H\left(\dot{\gamma}, Y^{*}\right), J H(\dot{\gamma}, \dot{\gamma})\right\rangle \alpha_{4}=0,
\end{aligned}
$$

where $Y^{\bullet}$ is the parallel vector field along $\gamma_{X}$ such that $Y^{\cdot}(0)=Y$. If

$$
\left\langle J Y^{\cdot}, H(\dot{\gamma}, \dot{\gamma})\right\rangle \neq 0 \quad \text { at some } t \in I,
$$

then $\alpha_{4}(t)=0$ by the fifth equation above and it is not difficult to derive $\alpha_{1}(t)=$ $\cdots=\alpha_{5}(t)$ from the other equations. Therefore we see that $\left\langle J Y^{*}, H(\dot{\gamma}, \dot{\gamma})\right\rangle \equiv 0$ on $I$, from which

$$
\langle J H(X, Y), H(X, X)\rangle+\left\langle J Y,(D H)\left(X^{3}\right)\right\rangle=0 .
$$

Suppose that $\left\langle J Y,(D H)\left(X^{3}\right)\right\rangle \neq 0$. Then by $\langle J Y, H(X, X)\rangle=0$ and (2.13), we have $\alpha_{1}(0)=\alpha_{4}(0)=\alpha_{5}(0)=0$ and the above seven equations reduce to

$$
\begin{gathered}
\alpha_{2}(0)+\langle J X, H(X, X)\rangle \alpha_{3}(0)=0, \\
\langle J X, H(X, X)\rangle \alpha_{2}(0)+\lambda^{2} \alpha_{3}(0)=0 .
\end{gathered}
$$

Since $\left(\alpha_{2}(0), \alpha_{3}(0)\right) \neq 0,\langle J X, H(X, X)\rangle= \pm \lambda$ which is equivalent to $H(X, X)=$ $\pm \lambda J X$. However we have a contradiction

$$
0 \neq\langle J H(X, Y), H(X, X)\rangle=\langle J H(X, Y), \pm \lambda J X\rangle=0 .
$$

We have proved $\langle J Y, H(X, X)\rangle=\left\langle J Y,(D H)\left(X^{3}\right)\right\rangle=0$. Q.E.D.

If $\mu=0$, the the second fundamental form is parallel. In the following two lemmas, we assume $\mu \neq 0$.

LEMMA 2.4. The function $\nu$ is defined on $U M$ is constant on each fiber $U_{x} M$.

ProOF. Since $\mu$ is constant, we have $\left\langle(D H)\left(X^{3}\right),\left(D^{2} H\right)\left(X^{4}\right)\right\rangle=0$ for every unit tangent vector $X$. By (2.4), it is easy to show

$$
\left\|\left(D^{2} H\right)\left(X^{4}\right)\right\|^{2}=\lambda^{2} \mu^{2}\left(\mu^{2}+\nu^{2}(X)\right) .
$$

Thus it suffices to prove that the derivative of $\left\|\left(D^{2} H\right)\left(X^{4}\right)\right\|^{2}$ in the direction $Y$ vanishes, where $X \in\{X \in U M ; \nu(X) \neq 0\}$ and $Y$ is a unit tangent vector orthogonal to $X$. Therefore we shall prove

$$
\left\langle\left(D^{2} H\right)\left(Y, X^{3}\right)+3\left(D^{2} H\right)\left(X^{3}, Y\right),\left(D^{2} H\right)\left(X^{4}\right)\right\rangle=0 .
$$

Suppose that

$$
\left\langle\left(D^{2} H\right)\left(Y, X^{3}\right),\left(D^{2} H\right)\left(X^{4}\right)\right\rangle=\left\langle\left(D^{2} H\right)\left(X^{3}, Y\right),\left(D^{2} H\right)\left(X^{4}\right)\right\rangle .
$$

We proved $\left\langle(D H)(X, X, Y),\left(D^{2} H\right)\left(X^{4}\right)\right\rangle=0$ in the proof of Lemma 2.3 (cf. (2.7) and (2.9)). It follows that

$$
\left\langle\left(D^{2} H\right)\left(X^{3}, Y\right),\left(D^{2} H\right)\left(X^{4}\right)\right\rangle+\left\langle(D H)(X, X, Y),\left(D^{3} H\right)\left(X^{5}\right)\right\rangle=0 .
$$


Since $(D H)(X, X, Y)$ is orthogonal to $\tau_{i}(X)$ for $i=1,2,3,4$, we have

$$
\left\langle\bar{\nabla}_{X}^{4} \dot{\tau}_{X},(D H)(X, X, Y)\right\rangle=0
$$

and hence $\left\langle(D H)(X, X, Y),\left(D^{3} H\right)\left(X^{5}\right)\right\rangle=0$. By (2.16) and (2.17), we obtain (2.15).

We must show (2.16). When $\bar{M}=\bar{M}(c)$, by the same argument as in the proof of Lemma 2.3, (2.16) can be proved. Thus suppose that $\bar{M}=C P^{q}(c)$ and $f$ is totally real. Then, from $(1.5)^{\prime}$ and (2.10),

$$
\begin{gathered}
\left\langle\left(D^{2} H\right)\left(Y, X^{3}\right)-\left(D^{2} H\right)\left(X, Y, X^{2}\right),\left(D^{2} H\right)\left(X^{4}\right)\right\rangle \\
=(c / 4)\left\{\langle J X, H(X, X)\rangle\left\langle J Y,\left(D^{2} H\right)\left(X^{4}\right)\right\rangle\right. \\
\left.-\langle J Y, H(X, X)\rangle\left\langle J X,\left(D^{2} H\right)\left(X^{4}\right)\right\rangle\right\} .
\end{gathered}
$$

Let $P$ be as before. Then the Frenet vectors $\tau_{1}(X), \ldots, \tau_{4}(X)$ of $\gamma_{X}$ span $T_{x} P$ at $x=\pi(X)$. If $P$ is totally real, then $\langle J X, H(X, X)\rangle=0=\left\langle J X,\left(D^{2} H\right)\left(X^{4}\right)\right\rangle$ and we have (2.16). Assume that $P$ is a complex submanifold in $C P^{q}(c)$. Since $\tau_{2}(X), \tau_{3}(X)$ and $\tau_{4}(X)$ are normal to $M,\left\langle Y, \tau_{i}(X)\right\rangle=0$ for $i=2,3,4$. Moreover $J H(X, X)$ and $J\left(D^{2} H\right)\left(X^{4}\right)$ are linear combinations of $\tau_{1}(X), \ldots, \tau_{4}(X)$. Thus $\langle Y, J H(X, X)\rangle=\left\langle Y, J\left(D^{2} H\right)\left(X^{4}\right)\right\rangle=0$. Substituting this equation into the righthand side of (2.18), we obtain (2.16). Q.E.D.

LEMMA 2.5. The function $\nu$ is constant on $M$.

PrOOF. Similarly, it suffices to show

$$
\left\langle\left(D^{3} H\right)\left(Y, X^{4}\right),\left(D^{2} H\right)\left(X^{4}\right)\right\rangle=0
$$

for every $X \in \pi^{-1}\left(S_{3}\right)$ and $Y$ orthogonal to $X$, where $S_{3}=\{x \in M ; \nu(x) \neq 0\}$. The same argument as in the proof of Lemma 2.4 implies

$$
\left\langle\left(D^{3} H\right)\left(Y, X^{4}\right)-\left(D^{3} H\right)\left(X, Y, X^{3}\right),\left(D^{2} H\right)\left(X^{4}\right)\right\rangle=0
$$

in both cases $\bar{M}=\bar{M}(c)$ and $C P^{q}(c)$. We proved $\left\langle\left(D^{2} H\right)\left(Y, X^{3}\right),\left(D^{2} H\right)\left(X^{4}\right)\right\rangle=0$ for any $X \in \pi^{-1}\left(S_{3}\right)$ and $Y$ orthogonal to $X$. Noting that $S_{3}$ is open, we get

$$
\left\langle\left(D^{3} H\right)\left(X, Y, X^{3}\right),\left(D^{2} H\right)\left(X^{4}\right)\right\rangle+\left\langle\left(D^{2} H\right)\left(Y, X^{3}\right),\left(D^{2} H\right)\left(X^{5}\right)\right\rangle=0 .
$$

Therefore we have only to prove $\left\langle\left(D^{2} H\right)\left(Y, X^{3}\right),\left(D^{3} H\right)\left(X^{5}\right)\right\rangle=0$ because of $(2.20)$. Since

$$
\left\langle\left(D^{2} H\right)\left(Y, X^{3}\right), H(X, X)\right\rangle=-\left\langle(D H)\left(X^{3}\right),(D H)(Y, X, X)\right\rangle=0,
$$

it follows from (2.7) and (2.16) that $\left(D^{2} H\right)\left(Y, X^{3}\right)$ is orthogonal to $\tau_{i}(X)$ for $i=$ 1, 2,3, 4. Therefore $\left\langle\bar{\nabla}_{X}^{4} \dot{\tau}_{X},\left(D^{2} H\right)\left(Y, X^{3}\right)\right\rangle=0$ and so (2.19) holds. Q.E.D.

By Lemmas 2.2-2.5, we have

THEOREM 2.6. Let $f: M \rightarrow \bar{M}(c)$ be a constant isotropic, 4-planar geodesic immersion into a real space form $\bar{M}(c)$. Then $f$ is a helical immersion of order $\leq 4$.

THEOREM 2.7. Let $f: M \rightarrow C P^{q}(c)$ be a constant isotropic, 4-planar geodesic, totally real immersion. Then $f$ is a helical immersion of order $\leq 4$.

For the definition of helical immersions, see [20]. 
3. Classification. In this section, we shall classify constant isotropic, 4-planar geodesic immersions into $S^{m}(1)$ and totally real, constant isotropic, 4-planar geodesic immersions into $C P^{q}(4)$. By the results of the preceding section, we have four cases:
(A) $\lambda=0$,
(B) $\lambda \neq 0, \mu=0$,
(C) $\lambda \mu \neq 0, \nu=0$,
(D) $\lambda \mu \nu \neq 0$.

Firstly, let the ambient manifold $\bar{M}$ be a Euclidean sphere $S^{m}(1)$ of constant curvature 1 . We assume that $M$ is connected and complete. In case (A), $f$ is totally geodesic and $M=S^{n}(1)$. In case (B), we see that the second fundamental form is parallel and $f$ is 2-planar geodesic. In [19], we classified all 2-planar geodesic immersions into $S^{m}(1)$. Next, let us consider cases (C) and (D) where the immersions are helical of orders 3 and 4 , respectively. In [14, 21 and 22], helical minimal immersions of orders 3 and 4 into $S^{m}(1)$ were determined. In the following, we exclude the minimality from the assumption and generalize the results obtained in those papers.

We recall some results about helical immersions (for detail, see [20 and 21]). Let $f: M \rightarrow S^{m}(1)$ be a helical immersion of order 3 or 4 . Then $\phi=\iota \circ f: M \rightarrow E^{m+1}$ is a helical immersion of order 4 , where $\iota$ denotes the canonical inclusion of $S^{m}(1)$ into $E^{m+1}$ whose origin coincides with the center. Let $\lambda, \mu$ and $\nu$ (resp. $\lambda_{1}, \lambda_{2}$ and $\lambda_{3}$ ) be the first, second and third Frenet curvatures of $f \circ \gamma($ resp. $\phi \circ \gamma)$ for each geodesic $\gamma$ of $M$, respectively. Then we see that

$$
\lambda_{1}=\left(1+\lambda^{2}\right)^{1 / 2}, \quad \lambda_{2}=\lambda \mu / \lambda_{1}, \quad \lambda_{3}=\left(\mu^{2}+\nu^{2}-\lambda^{2}\right)^{1 / 2},
$$

where $\nu=0$ if the order of $f$ is equal to 3 . The Frenet frame $\left\{\tau_{i}(X)\right\}, i=1, \ldots, 4$, of $\tau_{X}=\phi \circ \gamma_{X}$ at $x=\pi(X)$ is given by

$$
\begin{aligned}
& \tau_{1}(X)=X, \quad \tau_{2}(X)=\left(1 / \lambda_{1}\right)\{-x+H(X, X)\}, \\
& \tau_{3}(X)=\left(1 / \lambda_{1} \lambda_{2}\right)(D H)\left(X^{3}\right), \\
& \tau_{4}(X)=\left(1 / \lambda_{1} \lambda_{2} \lambda_{3}\right)\left\{-\lambda^{2} x+\lambda^{2} H(X, X)+\left(D^{2} H\right)\left(X^{4}\right)\right\},
\end{aligned}
$$

where we note that $\left(D^{2} H\right)\left(X^{4}\right)=-\mu^{2} H(X, X)$ if the order of $f$ equals 3 and $H$ is the second fundamental form of $f$. Define $f_{1}, \ldots, f_{4}$ by the differential equation

$$
\begin{aligned}
& f_{1}^{\prime}=1-\lambda_{1} f_{2}, \quad f_{2}^{\prime}=\lambda_{1} f_{1}-\lambda_{2} f_{3}, \\
& f_{3}^{\prime}=\lambda_{2} f_{2}-\lambda_{3} f_{4}, \quad f_{4}^{\prime}=\lambda_{3} f_{3}
\end{aligned}
$$

with initial conditions $f_{1}(0)=\cdots=f_{4}(0)=0$. Moreover define $\xi(s ; X)$ and $\varsigma(s ; X)$ by

$$
\begin{aligned}
& \xi(s ; X)=f_{2}(s) \tilde{\tau}_{2}(X)+f_{4}(s) \tilde{\tau}_{4}(X), \\
& \varsigma(s ; X)=f_{3}(s) \tau_{3}(X),
\end{aligned}
$$

where $\tilde{\tau}_{i}(X)=\tau_{i}(X)-\left\langle\tau_{i}(X), x\right\rangle x(i=2,4)$.

LEMMA 3.1 (CF. [20, 23]). Let $f: M \rightarrow S^{m}(1)$ be a helical immersion of a connected complete Riemannian manifold $M$. Then $M$ is a Blaschke manifold. If 
$f$ is not injective, then $M$ is isometric to a sphere $S^{n}$ and $f$ is the composite of the covering map $S^{n} \rightarrow R P^{n}$ and a helical imbedding $R P^{n} \rightarrow S^{m}(1)$.

Then the cut locus of every point is spherical (this fact is equivalent to the definition of a Blaschke manifold) and we denote by $L$ the diameter. Furthermore, we may asume that $f$ is an imbedding.

Lemma 3.2 (CF. [21]). Define $\xi_{X}(s ; V)$ and $(D \xi)(s ; V ; X)$ by

$$
\begin{gathered}
\xi_{X}(s ; V)=(d / d \theta) \xi(s ; \cos \theta X+\sin \theta(V /\|V\|))_{\theta=0}, \\
(D \xi)(s ; V ; X)=f_{2}(s)\left(D \tilde{\tau}_{2}\right)(V ; X)+f_{4}(s)\left(D \tilde{\tau}_{4}\right)(V ; X)
\end{gathered}
$$

for every $V$ orthogonal to $X \in U M$. Define $\varsigma_{X}(s ; V)$ and $\left(D_{\zeta}\right)(s ; V ; X)$ in a similar manner. Then

$$
\begin{aligned}
& \left(f_{1}(s)\right)^{2} H(V, X)+f_{1}(s)\left(D_{\zeta}\right)(s ; V ; X) \\
& \quad=F(s) \xi_{X}(s ; V)-\xi_{X}\left(s ; A_{\xi(s ; X)} V\right)-{ }\left(s ; A_{\zeta(s ; X)} V\right),
\end{aligned}
$$

where $F=1-\lambda_{1}^{-1} f_{2}-\lambda_{2}\left(\lambda_{1} \lambda_{3}\right)^{-1} f_{4}$.

LEMMA $3.3[\mathbf{2 1}]$. Let $\operatorname{Cut}(x)$ denote the cut locus of $x, b=F(L)$ and $a=$ $f_{1}^{\prime}(L)$. Let $X \in U_{x} M$. Then

(1) $\tau_{X}(s)=F(s) x+f_{1}(s) X+\xi(s ; X)+\varsigma(s ; X)$,

(2) $\operatorname{Cut}(x)=\left\{b x+\xi(L ; V) ; V \in U_{x} M\right\}$,

(3) $f_{1}(L)=f_{3}(L)=0,\left(f_{1}^{\prime}\right)^{2}+\left(f_{3}^{\prime}\right)^{2}=1, F^{\prime}=-f_{1}$,

(4) $A_{\xi(L ; X)} V=b V$ for every $v \in\{X\}^{\perp}$ if $a=0$,

(5)

$$
\begin{aligned}
& \left\langle\xi_{X}(s ; V), H(X, X)\right\rangle=\langle\xi(s ; X), H(X, V)\rangle=0, \\
& \left\langle\varsigma_{X}(s ; V), H(X, X)\right\rangle=\langle\varsigma(s ; X), H(X, V)\rangle=0 \text { for any } V \in\{X\}^{\perp}, \\
& \langle\xi(L ; X), H(X, X)\rangle=b-a,\langle\varsigma(s ; X), H(X, X)\rangle=0,
\end{aligned}
$$

where we identified $M$ with $\phi(M) \subset E^{m}$.

Making use of the above three lemmas, we prove

LEMMA 3.4. Let $f: M \rightarrow S^{m}(1)$ be a helical imbedding of a connected, complete Riemannian manifold $M$. If the order equals 3 , then $M$ is isometric to a Euclidean sphere $S^{n}$. If the order equals 4 , then $M$ is isometric to one of $S^{n}, R P^{n}, C P^{n / 2}, Q P^{n / 4}$ or Cay $P^{2}$ with canonical metric.

PROOF. Put $a_{3}=f_{3}^{\prime}(L)$. From (3) of Lemma 3.3, $a^{2}+a_{3}^{2}=1$. Suppose that the order equals 3. Assume $a \neq 0$. By the same argument as Theorem 4.1 of [21], $a_{3}=0$. Thus $a= \pm 1$. If $a=1$, then (3.1) implies that $f_{2}(L)=f_{4}(L)=0$ and hence $b=1$ which contradicts the injectivity of $f$. Noting that $\left(1 / \lambda_{1}\right)^{2}+\left(\lambda_{2} / \lambda_{1} \lambda_{3}\right)^{2}=1$ (cf. p. 74, Remark [20]) and using (3.1), we conclude $a=b=-1$. From (2) of Lemma 3.3 , it follows that $\operatorname{Cut}(x)$ consists of one point for every $x$. Thus, by the well-known Berger theorem (for exmple, see [2]), $M$ is isometric to a sphere. Assume $a=0$. Differentiate both sides of (3.2) twice at $s=L$. Using (3) and (4) in Lemma 3.3 , we have $\xi_{X}\left(L ; A_{\xi^{\prime \prime}(L ; X)} V\right)=-2 \zeta_{X}^{\prime}\left(L ; A_{\zeta^{\prime}(L ; X)} V\right)$ for $V \in\{X\}^{\perp}$. Take the inner product of both sides and $H(X, V)$. Then $A_{\varsigma^{\prime}(L ; X)} V=0$ because of (5) of Lemma 3.3 , from which $\xi_{X}\left(L ; A_{\xi^{\prime \prime}(L ; X)} V\right)=0$ for every $V \in\{X\}^{\perp}$. 
If $\xi(L ; X)=0$, then $b=-1$ which contradicts (4) of Lemma 3.3. Thus we see that $\xi(L ; X)=\alpha H(X, X)$ for some nonzero $\alpha$ and every $X \in U_{x} M$. Moreover, differentiating (1) of Lemma 3.3 twice at $s=L$,

$$
H\left(\dot{\gamma}_{X}(L), \dot{\gamma}_{X}(L)\right)-\tau_{X}(L)=\xi^{\prime \prime}(L ; X) .
$$

Thus $\xi^{\prime \prime}(L ; X) \neq 0$ and so $\xi^{\prime \prime}(L ; X)=\beta \xi(L ; X)$ for some nonzero $\beta$. Since $\langle\xi(L ; X), H(X, X)\rangle=\alpha \lambda^{2}$, we have $b \neq 0$. Using $(4), \xi_{X}(V)=0$ for every $V \in\{X\}^{\perp}$. This means that $\xi(X)$ is independent of $X \in U_{x} M$. We have proved again that $\operatorname{Cut}(x)$ consists of one point for every $x$.

Next, suppose that the order equals 4 . If $a \neq 0$, then we can apply the same argument as [21 and 22] to the (not necessarily minimal) helical immersions of order 4. (See Theorem 5.7, p. 786 [21] for the case $a>0$ and $\S 4$ of [22] for the case $a<0$. In those papers, the minimality of the immersion was essentially used to prove $a \neq 0$.) In the case $a \neq 0$, we see that $M$ is locally symmetric and hence it is a compact globally symmetric space of rank one since $M$ is a Blaschke manifold. Assume $a=0$. We have $A_{\zeta^{\prime}(L ; X)}=0$ by the same argument as the case where the order equals 3. Since $a_{3}= \pm 1$ and $\zeta^{\prime}(L ; X)=\left(\lambda_{1} \lambda_{2}\right)^{-1} a_{3}(D H)\left(X^{3}\right), A_{(D H)\left(X^{3}\right)}=$ 0 for every $X \in U M$. Making use of Gauss and Codazzi's equations, we conclude that $M$ is a locally symmetric space. Q.E.D.

Next, we explain the $i$ th standard minimal immersions and helical immersions constructed by Tsukada [24] (cf. $[2,7])$. Let $(M, g)$ be a compact symmetric space of rank one. Let $V_{i}$ be the $i$ th eigenspace of the Laplace operator $\Delta$ on $M, \mu_{i}$ the $i$ th eigenvalue and $\operatorname{dim} V_{i}=m(i)+1$. Define an inner product $\langle$,$\rangle on V_{i}$ by $\langle\phi, \psi\rangle=\int_{M} \phi \psi d v$, where $d v$ denotes the volume element of $M$. Taking an orthonormal base $\left\{\phi_{0}, \ldots, \phi_{m(i)}\right\}$ in $V_{i}$, we define a map $\Phi_{i}: M \rightarrow E^{m(i)+1}$ by $\Phi_{i}(x)=c_{i} \cdot\left(\phi_{0}(x), \ldots, \phi_{m(i)}(x)\right)$, where $c_{i}=\{\operatorname{vol} M /(m(i)+1)\}^{1 / 2}$. Then $\Phi_{i}$ becomes a full, helical minimal immersion of $\left(M,\left(\mu_{i} / n\right) g\right)$ into a unit hypersphere in $E^{m(i)+1}$, which is called the $i$ th standard minimal immersion into $S^{m(i)}(1)$. For distinct nonnegative integers $i_{1}, \ldots, i_{r}$ and positive real numbers $\alpha_{1}, \ldots, \alpha_{r}$ such that $\sum \alpha_{k}^{2}=1$, define $\Phi_{i_{1}, \ldots, i_{r} ; \alpha_{1}, \ldots, \alpha_{r}}$ by

$$
\Phi_{i_{1}, \ldots, i_{r} ; \alpha_{1}, \ldots, \alpha_{r}}(x)=\left(\alpha_{1} \Phi_{i_{1}}(x), \ldots, \alpha_{r} \Phi_{i_{r}}(x)\right) \in S^{m\left(i_{1}, \ldots, i_{r}\right)}(1),
$$

where $m\left(i_{1}, \ldots, i_{r}\right)=\sum m(k)+r-1$. Then this map becomes a helical immersion of $\left(M, g^{\prime}\right)$, where $g^{\prime}=\left\{\left(\sum \alpha_{k}^{2} \mu_{i_{k}}\right) / n\right\} g$. Tsukada [24] proved that if $f$ is a full helical immersion of a compact symmetric space of rank one into a unit sphere, then there exist $i_{1}, \ldots, i_{r}$ and $\alpha_{1}, \ldots, \alpha_{r}$ such that $f$ is congruent to $\Phi_{i_{1}, \ldots, i_{r} ; \alpha_{1}, \ldots, \alpha_{r}}$ (that is, there is an isometry $\Psi$ of the unit sphere such that $f=\Psi \circ \Phi_{i_{1}, \ldots, i_{r} ; \alpha_{1}, \ldots, \alpha_{r}}$ ). See also [7]. Tsukada computed the order of $\Phi_{i_{1}, \ldots, i_{r} ; \alpha_{1}, \ldots, \alpha_{r}}$ of compact symmetric spaces of rank one except for Cayley projective plane. Using the argument developed in [10], we easily see that his result is also true for a Cayley projective plane.

LEMMA 3.5 [24]. When $M=S^{n}$, the order of $\Phi_{i_{1}, \ldots, i_{r} ; \alpha_{1}, \ldots, \alpha_{r}}$ is equal to $\max \left\{i_{1}, \ldots, i_{r}\right\}$ if $i_{1}, \ldots, i_{r}$ are all odd or all even, and

$$
1+\max \left\{\text { odd numbers in } i_{1}, \ldots, i_{r}\right\}+\max \left\{\text { even numbers in } i_{1}, \ldots, i_{r}\right\}
$$

if $\left\{i_{1}, \ldots, i_{r}\right\}$ contains both odd numbers and even numbers. When $M=R P^{n}$, $C P^{n / 2}, Q P^{n / 4}$ or Cay $P^{2}$, the order of $\Phi_{i_{1}, \ldots, i_{r} ; \alpha_{1}, \ldots, \alpha_{r}}$ is equal to $2 \times$ $\max \left\{i_{1}, \ldots, i_{r}\right\}$. 
Using Lemmas 3.4 and 3.5, we obtain

THEOREM 3.6. Let $f: M \rightarrow S^{m}(1)$ be a constant isotropic, 4-planar geodesic immersion of a connected, complete Riemannian manifold $M$. Assume that $f$ is full. For convenience sake, we abbreviate $\Phi_{i_{1}, \ldots, i_{r} ; \alpha_{1}, \ldots, \alpha_{r}}$ as $\left\{i_{1}, \ldots, i_{r}\right\}$, where $\alpha_{k}$ 's are determined by the immersion $f$. Then we have

(A) $M=S^{n}, f \approx\{1\}$,

(B) $M=S^{n}, f \approx\{2\},\{0,1\}$ or $\{0,2\}, M=F P^{n / e}, f \approx\{1\}$ or $\{0,1\}$,

(C) $M=S^{n}, f \approx\{3\}$ or $\{1,3\}$,

(D)

$$
\begin{aligned}
& M=S^{n}, \\
& f \approx\{4\},\{0,3\},\{0,4\},\{1,2\},\{2,4\},\{0,1,2\},\{0,1,3\} \text { or }\{0,2,4\}, \\
& M=F P^{n / e}, f \approx\{2\},\{0,2\},\{1,2\} \text { or }\{0,1,2\},
\end{aligned}
$$

where $e=1,2,4$ or 8 according as $F=R, C, Q$ or Cay.

Secondly, let $f: M \rightarrow C P^{q}(4)$ be a constant isotropic, 4-planar geodesic, totally real immersion of a connected, complete Riemannian manifold. We shall prove that $f(M)$ is contained in a totally real, totally geodesic submanifold of $C P^{q}(4)$ in cases (C) and (D).

LEMMA 3.7. If $f$ is a helical immersion of order 3 (resp. 4), then, for each geodesic $\gamma$ of $M$, there exists a unique 3 (resp. 4)-dimensional, totally real, totally geodesic submanifold $P_{\gamma}$ such that $\tau((-\infty, \infty)) \subset P_{\gamma}$, where $\tau=f \circ \gamma$.

ProOF. Let $f$ be a helical immersion of order 3 (i.e., case $(\mathrm{C})$ ). Define $\Pi_{X}$ by $\Pi_{X}=\operatorname{Sp}\left\{\tau_{i}(X), J \tau_{i}(X): i=1,2,3\right\} \subset T_{\pi(X)} C P^{q}(c)$ for $X \in U M$. Since $\tau_{i}(X)$ 's are orthonormal and $\Pi_{X}$ is a complex subspace, $\operatorname{dim} \Pi_{X}=4$ or 6 . Let $\gamma_{X}$ be the unit speed geodesic such that $\gamma_{X}(0)=\pi(X)$ and $\dot{\gamma}_{X}(0)=X$. By the assumption, there exist a 4-dimensional totally geodesic submanifold $P_{X}$ and an open interval $I(\ni 0)$ such that $\tau_{X}(I) \subset P_{X}$, where $\tau_{X}=f \circ \gamma_{X}$. Such $P_{X}$ is not necessarily unique. Denote by $\Gamma_{X}$ the set of $P_{X}$ 's. Here, we note that every totally geodesic submanifold of $C P^{q}(c)$ is complex or totally real because of $(1.4)^{\prime}$. Since the intersection of a 4-dimensional complex totally geodesic submanifold and totally real totally geodesic submanifold is a totally geodesic submanifold of dimension $\leq 2$ and $\mu \neq 0$, if some element of $\Gamma_{X}$ is complex, then $\Gamma_{X}$ consists of one complex totally geodesic submanifold $P_{X}$. Since $\tau_{i}(X)$ 's are tangent to $P_{X}$, if $\Gamma_{X}$ contains a totally real $P_{X}$, then $\operatorname{dim} \Pi_{X}=6$, and if $\Gamma_{X}$ contains a complex $P_{X}$, then $\operatorname{dim} \Pi_{X}=4$. Therefore we see that

$$
\begin{aligned}
& \left\{X \in U M ; \operatorname{dim} \Pi_{X}=6\right\} \\
& \quad=\left\{X \in U M ; \operatorname{Sp}\left\{\tau_{1}(X), \tau_{2}(X), \tau_{3}(X)\right\} \text { is totally real }\right\} .
\end{aligned}
$$

It is easy to show that the left-hand side is open in $U M$. On the other hand, the right-hand side is closed since it is characterized by equations $\left\langle J \tau_{i}(X), \tau_{j}(X)\right\rangle=$ $0(i, j=1,2,3)$. Thus if $\left\{X \in U M ; \operatorname{dim} \Pi_{X}=6\right\} \neq \varnothing$, then this set coincides with $U M$. We have shown that every element of $\bigcup_{X \in U M} \Gamma_{X}$, i.e., every $P_{X}$ is complex for all $X \in U M$ or totally real for all $X \in U M$. Assume that $P_{X}$ is complex for all $X$. Then by the same argument as in the proof of Lemma 2.3, 
$\langle J Y, H(X, X)\rangle=0$ for all orthonormal vectors $X$ and $Y$. Put $X(\theta)=\cos \theta X+$ $\sin \theta Y$. Differentiating $\left\langle J X^{\prime}(\theta), H(X(\theta), X(\theta))\right\rangle=0$ at $\theta=0$ and using (1.6), we have $\langle J X, H(X, X)\rangle=0$ for every $X \in U M$. It follows from (1.1), (1.2) and (1.8) that $\left\langle J X,(D H)\left(X^{3}\right)\right\rangle=0$ for every $X$. Again from (1.1), (1.2), (2.1) and $\left(D^{2} H\right)\left(X^{4}\right)=-\mu^{2} H(X, X)$, we obtain $\left\langle J H(X, X),(D H)\left(X^{3}\right)\right\rangle=0$. Therefore $\operatorname{dim} \Pi_{\chi}=6$, which is a contradiction. Thus every $P_{X}$ is totally real. Since $P_{X}$ is isometric to $R P^{4}(1)$ and $\nu=0$, there is a 3-dimensional totally real, totally geodesic submanifold $P$ and an open interval $I$ ( $\ni 0$ ) such that $\tau_{X}(I) \subset P \subset P_{X}$. Noting that $\mu \neq 0$ and the intersection of two 3-dimensional, totally real, totally geodesic submanifolds is a totally real, totally geodesic submanifold of dimension $\leq 2$, we see that for each geodesic $\gamma$ there exists a unique 3-dimensional, totally real, totally geodesic submanifold $P_{\gamma}$ such that $\tau((-\infty, \infty)) \subset P_{\gamma}$.

Next, let $f$ be a helical immersion of order 4 (i.e., case (D)). The existence and uniqueness of $P_{\gamma}$ follow from the assumption that $f$ is 4-planar geodesic and $\nu \neq 0$ (cf. Lemma 2.1 of [18]). Assume that there exist $X, Y \in U M$ such that $P_{\gamma_{X}}$ is complex and $P_{\gamma_{Y}}$ is totally real. Take a smooth curve $X(t)$ in $U M$ that satisfies $X(0)=X$ and $X(1)=Y$. Consider a function $u$ on $[0,1]$ defined by

$$
u(t)=\operatorname{Sup}\left\{\langle J X(t), Z\rangle ; Z \in T_{\pi(X(t))} P_{\gamma_{X(t)}},\|Z\|=1\right\} .
$$

The tangent space of $P_{\gamma_{X(t)}}$ at $\pi(X(t))$ is spanned by $\tau_{1}(X(t)), \ldots, \tau_{4}(X(t))$ which vary smoothly and hence it is a smooth curve in the Grassmann bundle of 4-planes over $M$. Thus $u$ is a continuous function. Since every $P_{\gamma_{X(t)}}$ is complex or totally real, $u(t)=1$ or 0 for each $t \in[0,1]$. It follows that $u$ is contant. However $u(0)=1$ and $u(1)=0$. We have shown that $P_{\gamma}$ is complex for every geodesic $\gamma$ or totally real for every $\gamma$. Assume that $P_{\gamma_{X}}$ is complex for every $X \in U M$. Since $J X$ is a linear combination of $\tau_{2}(X), \tau_{3}(X)$ and $\tau_{4}(X)$, if $Y$ is orthonormal to $X$, then $\langle J X, H(X, Y)\rangle=0$ by means of (1.8), (2.1) and (2.6). Differentiating $\left\langle J X(\theta), H\left(X(\theta), X^{\prime}(\theta)\right)\right\rangle=0$ at $\theta=0$, we get $\langle J Y, H(X, Y)\rangle+\langle J X, H(Y, Y)\rangle-$ $\langle J X, H(X, X)\rangle=0$. By (1.6), $\langle J X, H(X, X)\rangle=0$ for every $X \in U M$. Also we have $\left\langle J X,(D H)\left(X^{3}\right)\right\rangle=0$ in the same way as the case $(\mathrm{C})$. Thus $\left\langle J X, \tau_{4}(X)\right\rangle= \pm 1$ on $U M$. Since $n \geq 2$ and the left-hand side is the restriction to $U_{x} M$ of a polynomial of degree 5, we have a contradiction. Q.E.D.

LEMMA 3.8. Let $f: M \rightarrow C P^{q}(4)$ be a totally real immersion. If, for every $X \in T M$, and $i, j \in\{1, \ldots, d\}$,

$$
\left\langle J\left(D^{i-2} H\right)\left(X^{i}\right),\left(D^{j-2} H\right)\left(X^{j}\right)\right\rangle=0,
$$

then the subspace $O_{d}(x)$ defined by

$$
O_{d}(x)=\operatorname{Sp}\left\{\left(D^{i-2} H\right)\left(X_{1}, \ldots, X_{i}\right): i=1, \ldots, d, X_{1}, \ldots, X_{i} \in T_{x} M\right\}
$$

is totally real in $T_{x} C P^{q}(4)$, where $x$ is arbitrarily fixed and $\left(D^{-1} H\right)(X)$ (resp. $\left.\left(D^{0} H\right)(X, Y)\right)$ is understood as $\left(D^{-1} H\right)(X)=X\left(\right.$ resp. $\left.\left(D^{0} H\right)(X, Y)=H(X, Y)\right)$.

ProOF. We prove

$$
\left\langle J\left(D^{i-2} H\right)\left(X_{1}, \ldots, X_{i}\right),\left(D^{j-2} H\right)\left(Y_{1}, \ldots, Y_{j}\right)\right\rangle=0 \quad(1 \leq i, j \leq d),
$$

for every $X_{1}, \ldots, X_{i}, Y_{1}, \ldots, Y_{j} \in T_{x} M$ by the induction with respect to $r=i+j$. If $r=2$, then (3.4) holds since $\langle J X, Y\rangle=0$. Assume that (3.4) holds for every $i, j$ 
such that $r$ is not greater than $p(<2 d)$. Let $r=p+1(1 \leq i, j \leq p)$. We regard the left-hand side of (3.4) as a multilinear $(p+1)$-form. From (3.3), we have only to show that this form is symmetric. If $j \geq 4$, then Ricci's identity

$$
\begin{aligned}
\left(D^{h} H\right) & \left(X, Y, X_{1}, \ldots, X_{h}\right)-\left(D^{h} H\right)\left(Y, X, X_{1}, \ldots, X_{h}\right) \\
= & R^{\perp}(X, Y)\left(D^{h-2} H\right)\left(X_{1}, \ldots, X_{h}\right) \\
& -\sum_{t}\left(D^{h-2} H\right)\left(X_{1}, \ldots, R(X, Y) X_{t}, \ldots, X_{h}\right),
\end{aligned}
$$

(1.4), (1.5)' and the assumption of the induction imply that

$$
\begin{gathered}
\left(D^{j-2} H\right)\left(Y_{1}, \ldots, Y_{k}, Y_{k+1}, \ldots, Y_{j}\right)-\left(D^{j-2} H\right)\left(Y_{1}, \ldots, Y_{k+1}, Y_{k}, \ldots, Y_{j}\right) \\
=\text { linear combination of } H(\cdot, \cdot), \ldots,\left(D^{j-4} H\right)(\cdot, \ldots, \cdot) .
\end{gathered}
$$

It follows from the assumption of the induction that for every $i, j$ with $i+j=p+1$ L.H.S. of (3.4) is symmetric with respect to every pair chosen from $\left\{Y_{1}, \ldots, Y_{j}\right\}$ and similarly, it is symmetric with respect to every pair chosen from $\left\{X_{1}, \ldots, X_{i}\right\}$. Thus it suffices to show that L.H.S. of (3.4) is symmetric with respect to $X_{1}$ and $Y_{1}$. Differentiate

$$
\left\langle J\left(D^{i-2} H\right)\left(X_{1}^{*}, \ldots, X_{i}^{*}\right),\left(D^{j-3} H\right)\left(Y_{2}^{*}, \ldots, Y_{j}^{*}\right)\right\rangle=0
$$

in the direction $Y_{1}$, where $X^{*}$ means the vector field such that $X^{\cdot}(x)=X$ and $\nabla X^{\cdot}=0$ at $x$. Then, using (1.1) and (1.2),

$$
\begin{aligned}
& \left\langle J\left(D^{i-1} H\right)\left(Y_{1}, X_{1}, \ldots, X_{i}\right),\left(D^{j-3} H\right)\left(Y_{2}, \ldots, Y_{j}\right)\right\rangle \\
& \quad+\left\langle J\left(D^{i-2} H\right)\left(X_{1}, \ldots, X_{i}\right),\left(D^{j-2} H\right)\left(Y_{1}, Y_{2}, \ldots, Y_{J}\right)\right\rangle=0 .
\end{aligned}
$$

Since $(i+1)+(j-1)=p+1$, the first term is symmetric with respect to $X_{1}$ and $Y_{1}$, and hence so is the second term. Q.E.D.

LEMMA 3.9. In the cases (C) and (D), there exists a totally real, totally geodesic submanifold $Q\left(=R P^{k}(1)\right)$ such that $f(M) \subset Q$ and $f: M \rightarrow Q$ is full.

ProOF. For each $X \in U M, \operatorname{Sp}\left\{\tau_{1}(X), \ldots, \tau_{d}(X)\right\}$ is totally real in virtue of Lemma 3.7, where $d=3$ or 4 according as (C) or (D). Thus (3.3) holds in cases (C) and (D). By Lemma $3.8 O_{d}(x)$ at a distinguished point $x$ is totally real in $T_{x} C P^{q}(4)$. So, we have a totally real, totally geodesic submanifold $Q$ tangent to $O_{d}(x)$ at $x$ (which is uniquely determined). Let $y$ be an arbitrary point of $M$ and $\gamma$ a unit speed geodesic connecting $x$ with $y$. Let $X=\dot{\gamma}(0) \in U_{x} M$. The curve $\tau=f \circ \gamma$ satisfies the Frenet equation

$$
\begin{array}{cc}
\dot{\tau}=\tau_{1}, \quad \bar{\nabla}_{\dot{\gamma}} \tau_{1}=\kappa_{1} \tau_{2}, \quad \bar{\nabla}_{\dot{\gamma}} \tau_{i}=-\kappa_{i-1} \tau_{i-1}+\kappa_{i} \tau_{i+1} \quad(2 \leq i \leq d-1), \\
\bar{\nabla}_{\dot{\gamma}} \tau_{d}=-\kappa_{d-1} \tau_{d-1},
\end{array}
$$

where we have put $\kappa_{1}=\lambda, \kappa_{2}=\mu$ and $\kappa_{3}=\nu$. The initial conditions are $\tau(0)=x$ and $\tau_{i}(0)=\tau_{i}(X)(i=1, \ldots, d)$ which are contained in $O_{d}(x)$. Consider a curve $\omega$ in $Q$ whose $i$ th curvature is $\kappa_{i}$ for $i=1, \ldots, d$ and which satisfies $\omega(0)=x, \omega_{i}(0)=$ $\tau_{i}(X)(i=1, \ldots, d)$, where $\omega_{i}$ is the $i$ th Frenet vector of $\omega$. Since $Q$ is totally geodesic, the fundamental theorem of ordinary differential equation implies $\tau=\omega$. Thefore $f(y) \in Q$. It is easily verified that $f: M \rightarrow Q$ is full. Q.E.D. 
We lift $f: M \rightarrow Q$ to $\hat{f}: M \rightarrow S^{k}(1)$ by the Riemannian covering $p: S^{k}(1) \rightarrow$ $R P^{k}(1)=Q$. Since $\hat{f}$ is also a full constant isotropic, 4-planar geodesic immersion of type (C) or (D), we can apply Theorem 3.6 to $\hat{f}$ and have the complete classification.

Next, we consider the case (B). In this case, the second fundamental form is parallel. Thus the classification reduces to that of isotropic, totally real submanifolds with parallel second fundamental forms, which was done in Naitoh [11] (see also $[12, \mathbf{1 3}])$. He showed that the following cases occur

$\left(\mathrm{B}_{1}\right) J T_{x} M \subset\left(N_{x}^{1}\right)^{\perp}$ for every $x \in M$,

$\left(\mathrm{B}_{2}\right) J T_{x} M \subset N_{x}^{1}$ for every $x \in M$,

where $N_{x}^{1}$ is the first normal space at $x$ defined by $N_{x}^{1}=\operatorname{Sp}\left\{H(X, Y) ; X, Y \in T_{x} M\right\}$. Since (3.3) holds in the case $\left(\mathrm{B}_{1}\right)$ and so $O_{2}(x)=T_{x} M+N_{x}^{1}$ is totally real, there exists a totally real, totally geodesic submanifold $Q$ such that $f(M) \subset Q$ and $f: M \rightarrow Q$ is full (by the same argument as Lemma 3.9). Thus case $\left(\mathrm{B}_{1}\right)$ reduces to $(\mathrm{B})$ in Theorem 3.6. It is easily verified in case $\left(\mathrm{B}_{2}\right)$ that there exists a complex, totally geodesic submanifold $N$ of $C P^{q}(4)$ such that $f(M) \subset N$ and $f: M \rightarrow N$ is full (cf. $[\mathbf{1 1}, \mathbf{1 2}])$.

LEMMA $3.10[\mathbf{1 1 - 1 3}]$. Let $M$ be an $n(\geq 2)$-dimensional complete, simply connected, totally real, isotropic submanifold of type $\left(\mathrm{B}_{2}\right)$ with parallel second fundamental form in $C P^{q}(4)$. Then $\operatorname{dim}_{C} N=n, M$ is isometric to one of the symmetric spaces; $R^{2}, R \times S^{n-1}(n \geq 3), \mathrm{SU}(3) / \mathrm{SO}(3), \mathrm{SU}(6) / \mathrm{Sp}(3), E_{6} / F_{4}$, and the immersion is rigid.

The concrete construction of totally real, isotropic, parallel immersions of the above symmetric spaces were also given in [11 and 12]. Here, we simply denote them by $\Phi$. It suffices to examine that $\Phi$ is 4 -planar geodesic. Since the above symmetric spaces are of rank two, for a given geodesic $\gamma$ of $M$, there is a 2-dimensional, flat, totally geodesic submanifold $F$ of $M$ such that $\gamma$ is contained in $F$. The restriction of $f$ to $F$ inherits the properties that $f$ is totally real, isotropic and the second fundamental form is parallel. Thus, by Lemma 3.10, the universal Riemannian covering manifold of $F$ is isometric to $R^{2}$ and $f(F)$ is contained in a complex 2-dimensional totally geodesic submanifold.

THEOREM 3.11. Let $f: M^{n} \rightarrow C P^{q}(4)$ be a constant isotropic, 4-planar geodesic, totally real immersion of a complete, simply connected Riemannian manifold $(n \geq 2)$. Let $\iota_{1}: R P^{k}(1) \rightarrow C P^{q}(4)\left(\right.$ resp. $\left.\iota_{2}: C P^{n}(4) \rightarrow C P^{q}(4)\right)$ be a totally real (resp. complex), totally geodesic immersion, where $k=\operatorname{dim} Q$. Then we see that

(A) $M=S^{n}, f \approx \iota_{1} \circ p(k=n)$,

$\left(\mathrm{B}_{1}\right) M=S^{n}, F P^{n / e}, f \approx \iota_{1} \circ p \circ$ (immersion in (B) of Theorem 3.6),

$\left(\mathrm{B}_{2}\right) M=$ symmetric spaces in Lemma $3.10, f \approx \iota_{2} \circ \Phi$,

(C) $M=S^{n}, f \approx \iota_{1} \circ p \circ$ (immersion in (C) of Theorem 3.6),

(D) $M=S^{n}, F P^{n / e}, f \approx \iota_{1} \circ p \circ$ (immersion in (D) of Theorem 3.6), where $F \neq R$.

4. An example of isotropic immersion $T^{2} \rightarrow S^{5}(1)$. In this section, we give an example of constant isotropic immersion of a torus $T^{2}$ into $S^{5}(1)$. It is 5 -planar geodesic, but not 4-planar geodesic. We show that the function $\mu$ defined by $\mu(X)=\left\|(D H)\left(X^{3}\right)\right\| / \lambda$ depends on $X \in U_{x} T^{2}$ (cf. Remark $\S 2$ ). This example was given in the study of stationary 2-type surfaces in a sphere (see, M. Barros and 
B.-Y. Chen [1]). Notice that we can not replace the assumption that $f$ is 4-planar geodesic by 5 -planar geodesic in Theorem 2.6 .

Let $f$ be a map from $E^{2}$ to $E^{6}$ defined by

$$
\begin{aligned}
& f(u, v)=(1 / \sqrt{6})(2 \cos x \cos y, 2 \cos x \sin y, \sqrt{2} \cos 2 x, \\
& 2 \sin x \cos y, 2 \sin x \sin y, \sqrt{2} \sin 2 x),
\end{aligned}
$$

where $(u, v) \in E^{2}$ and $x=u / \sqrt{2}, y=\sqrt{6} v / 2$. It is easily verified that $f\left(E^{2}\right) \subset$ $S^{5}(1)$ and $f$ is an isometric imbedding of a flat torus. Let $\gamma$ be a geodesic of $E^{2}$ which is given by

$$
u=\alpha s+\alpha_{0}, \quad v=\beta s+\beta_{0},
$$

where $s$ is the arc-length parameter and $\alpha^{2}+\beta^{2}=1$. Since $\tau(s)=(f \circ \gamma)(s)$ satisfies

$$
\ddot{\tau}=d^{2} f / d s^{2}=H(\dot{\gamma}, \dot{\gamma})-\tau,
$$

$H$ being the second fundamental form of the immersion $f: E^{2} \rightarrow S^{5}(1)$, we have $H(\dot{\gamma}, \dot{\gamma})=\ddot{\tau}+\tau$. Using (4.1), a straightforward computation shows

$$
\begin{aligned}
H(\dot{\gamma}, \dot{\gamma})=(1 / \sqrt{6})\{ & \left(1-\varepsilon^{2}\right) \cos (x+y)+\left(1-\delta^{2}\right) \cos (x-y), \\
& \left(1-\varepsilon^{2}\right) \sin (x+y)-\left(1-\delta^{2}\right) \sin (x-y), \\
& \sqrt{2}\left(1-2 \alpha^{2}\right) \cos 2 x,\left(1-\varepsilon^{2}\right) \sin (x+y) \\
& +\left(1-\delta^{2}\right) \sin (x-y),-\left(1-\varepsilon^{2}\right) \cos (x+y) \\
& \left.\quad+\left(1-\delta^{2}\right) \cos (x-y), \sqrt{2}\left(1-2 \alpha^{2}\right) \sin 2 x\right\},
\end{aligned}
$$

where $x=\left(\alpha s+\alpha_{0}\right) / \sqrt{2}, y=\sqrt{6}\left(\beta s+\beta_{0}\right) / 2, \varepsilon=(\sqrt{2} \alpha+\sqrt{6} \beta) / 2$ and $\delta=$ $(\sqrt{2} \alpha-\sqrt{6} \beta) / 2$. Therefore we have $\lambda^{2}=\|H(\gamma, \gamma)\|^{2}=1 / 2$, which is independent of $\gamma$. Noting that

$$
\dddot{\tau}+\dot{\tau}=-\lambda^{2} \dot{\tau}+(D H)(\dot{\gamma}, \dot{\gamma}, \dot{\gamma})
$$

from (4.2)

$$
\begin{aligned}
& (D H)(\dot{\gamma}, \dot{\gamma}, \dot{\gamma}) \\
& =(1 / \sqrt{3}) \alpha\left(4 \alpha^{2}-3\right)\{-\sin x \cos y,-\sin x \sin y,(1 / \sqrt{2}) \sin 2 x, \\
& \quad \cos x \cos y, \cos x \sin y,-(1 / \sqrt{2}) \cos 2 x\} .
\end{aligned}
$$

Therefore we see that the first normal space at each point is 2-dimensional and its orthogonal complement in the normal space is spanned by

$$
\{-\sin x \cos y,-\sin x \sin y,(1 / \sqrt{2}) \sin 2 x, \cos x \cos y, \cos x \sin y,-(1 / \sqrt{2}) \cos 2 x\} .
$$

Let $X=\alpha \partial / \partial u+\beta / \partial v$ at $\left(\alpha_{0}, \beta_{0}\right) \in E^{2}$. Then

$$
\mu(X)=2\left\|(D H)\left(X^{3}\right)\right\|=|\alpha|\left|4 \alpha^{2}-3\right| .
$$

It follows that $\mu(X)=0$ if and only if $\alpha=0$ or $\alpha= \pm \sqrt{3} / 2$. Finally, let $\left(\alpha_{0}, \beta_{0}\right)=$ $(0,0)$ and $\beta / \alpha=2 / \sqrt{3}$. Then $\alpha= \pm \sqrt{3} / \sqrt{7}$ and the image of the geodesic; $u=\alpha s$, $v=(2 \alpha / \sqrt{3}) s$ is given by

$(1 / \sqrt{6})(2 \cos x \cos 2 x, 2 \cos x \sin 2 x, \sqrt{2} \cos 2 x, 2 \sin x \cos 2 x$,

$$
2 \sin x \sin 2 x, \sqrt{2} \sin 2 x),
$$

where $x=\alpha s$. Since the coordinate components are linearly independent, this curve is not contained in any 5-plane through the origin of $E^{6}$. Thus it is not contained in any 4-dimensional great sphere of $S^{5}(1)$. 


\title{
REFERENCES
}

1. M. Barros and B.-Y. Chen, Stationary 2-type surfaces in a hypersphere, preprint.

2. A. Besse, Manifolds all of whose geodesics are closed, Ergebnisse Math., Band 93, Springer, Berlin and New York, 1978.

3. B.-Y. Chen, Geometry of submanifold, Dekker, New York, 1973.

4. B.-Y. Chen and K. Ogiue, On totally real submanifolds, Trans. Amer. Math. Soc. 193 (1974), 257-266.

5. D. Ferus, Symmetric submanifolds of Euclidean space, Math. Ann. 247 (1980), 81-93.

6. S. Helgason, Differential geometry, Lie groups and symmetric spaces, Academic Press, New York, 1978.

7. Y. Hong, Helical submanifolds in euclidean spaces, Indiana Univ. Math. J. 35 (1986), 29-43.

8. Y. Hong and C.-S. Hough, Helical immersions and normal sections, Kodai Math. J. 8 (1985), 171-192.

9. J. A. Little, Manifolds with planar geodesics, J. Differential Geom. 11 (1976), 265-285.

10. K. Mashimo, Order of the standard isometric immersions of CROSS as helical geodesic immersions, Tsukuba J. Math. 7 (1983), 257-263.

11. H. Naitoh, Isotropic submanifolds with parallel second fundamental form in $P^{m}(c)$, Osaka J. Math. 18 (1981), 427-464.

12. _ _ Totally real parallel submanifolds in $P^{n}(c)$, Tokyo J. Math. 4 (1981), 279-306.

13. H. Naitoh and M. Takeuchi, Parallel submanifolds in symmetric spaces, Sugaku 36 (1984), 137-156 (in Japanese).

14. H. Nakagawa, On a certain minimal immersion of a Riemannian manifold into a sphere, Kodai Math. J. 3 (1980), 321-340.

15. B. O'Neill, Isotropic and Kaehler immersions, Canad. J. Math. 17 (1965), 907-915.

16. J. S. Pak, Planar geodesic submanifolds in complex space forms, Kodai Math. J. 1 (1978), 187-196.

17. J. S. Pak and K. Sakamoto, Submanifolds with proper d-planar geodesics immersed in complex projective spaces, Tôhoku Math. J. 38 (1986), 297-311.

18. __ 4-planar geodesic Kaehler immersions into a complex projective space, preprint.

19. K. Sakamoto, Planar geodesic immersions, Tôhoku Math. J. 29 (1977), 25-56.

20. __ Helical immersions into a unit sphere, Math. Ann. 261 (1982), 63-80.

21. __ Helical minimal immersions of compact Riemannian manifolds into a unit sphere, Trans. Amer. Math. Soc. 288 (1985), 765-790.

22. __ Helical minimal immbeddings of order 4 into spheres, J. Math. Soc. Japan 37 (1985), 315-336.

23. __ Helical immersions into a Euclidean space, Michigan Math. J. 33 (1986), 353-364.

24. K. Tsukada, Helical geodesic immersions of compact rank one symmetric spaces into spheres, Tokyo J. Math. 6 (1983), 267-285.

\author{
DePaRTMENT OF MAThEMATICS, KYNGPOOK NATIONAL UNIVERSity, TAEGU, 702-701 \\ KOREA
}

Department of Mathematics, Tokyo instutite of Technology, Tokyo, 152 JAPAN 